\title{
CONCERNING THE ARC-CURVES AND BASIC SETS OF A CONTINUOUS CURVE*
}

\author{
BY \\ W. L. AYRES
}

In this paper we define and discuss some of the properties of certain subsets of a continuous curve, $\nmid$ which we call the arc-curves. Probably the most interesting property of these subsets is that whenever the arc-curve is closed, it is itself a continuous curve. The arc-curves are intimately connected with the theory of separation of points in continuous curves, since if a closed set separates a pair of points in the arc-curve of the pair of points with respect to a given continuous curve, the closed set separates the points in the given continuous curve. We define a basic set of a continuous curve as any set whose arc-curve is the continuous curve itself. Necessary and sufficient conditions are given in order that a subset of a continuous curve be a basic set and in order that a basic set be irreducible.

It is assumed that all point sets of the paper lie in a two-dimensional euclidean space. $\neq$

Notation. Wherever a symbol $H$ is used to denote a point set, the symbol $\vec{H}$ denotes the point set consisting of the points of $H$ plus all limit points of $H$. If $u$ and $v$ are points of a simple continuous arc $\alpha$, the symbol $u v$ denotes the subarc of $\alpha$ with $u$ and $v$ as end points if $u \neq v$, and if $u=v$, the symbol $u v$ denotes the single point $u(=v)$. Hereafter we shall use the term "arc" to mean "simple continuous arc." If $u v$ is an arc with end points $u$ and $v$, the symbols $\langle u v, u v\rangle,\langle u v\rangle$ denote $u v-u, u v-v, u v-u-v$ respectively. If $A$ and $B$ are sets of points, the symbol $A \subset B$ means $A$ is a subset of $B$, but it is not implied that $A$ is a proper subset of $B$.

\section{I}

Lemma. If $P$ is a point of a set $K$ such that $K-P$ is the sum of two mutually separated point sets $K_{1}$ and $K_{2}$ and $A$ and $B$ are distinct points of $K_{1}+P$, then $K_{2}$ contains no point of any arc from $A$ to $B$ which belongs entirely to $K$.

* Presented to the Society, May 7, 1927; received by the editors in July, 1927.

† A continuous curve is a point set which is connected and connected im kleinen. This is sometimes called a generalized continuous curve. See S. Mazurkiewicz, Sur les lignes de Jordan, Fundamenta Mathematicae, vol. 1 (1920), p. 193.

$\ddagger$ (Added in proof.) In a paper Concerning continuous curves in space of $n$ dimensions, which is not yet published, $I$ have recently shown that all the results of this paper, except part of Theorem 10 , hold in euclidean space of $n$ dimensions. 
Definition. Let $K$ be a subset of a continuous curve $M$. The set of all points $[P]$, such that $P$ lies on some arc of $M$ whose end points are points of $K$, will be called the arc-curve of $K$ with respect to $M$ and will be denoted by $M(K)$. In case $K$ consists of a single point, we define the arc-curve of $K$ with respect to $M$ to be this single point.

It is evident that the set $M(K)$ is connected and contains the set $K$. We shall proceed to determine further properties of the point set $M(K)$.

THEOREM 1. If $H$ and $K$ are subsets of the continuous curve $M$ and $B$ is a subset of $K$, then $M(H)$ is a subset of $M(K)$.

The truth of this theorem follows easily from the definition.

Theorem 2. If $K$ is any subset of the continuous curve $M$, the point set $\overline{M(K)}-M(K)$ is a subset of the point set $\bar{K}-K$.

Suppose there exists a point $x$ which belongs to $\overline{M(K)}-M(K)$ but not to $\bar{K}-K$. Then $x$ belongs to $M-\bar{K}$. Let $y$ be a point of $K$, and let $x y$ denote an $\operatorname{arc}$ of $M$ whose end points are $x$ and $y$. If $d$ is any component* of $M-x y$, let $P_{x d}$ and $P_{y d}$ denote the first and last limit points of $d$ on $x y$ in the order from $x$ to $y$. The points $P_{x d}$ and $P_{y d}$ exist (but are not necessarily distinct) for each component $d$ since the set of limit points of $d$ on the arc $x y$ is closed. Let $z_{1}$ denote the first point of $\bar{K}+y$ on the arc $x y$ in the order $x$ to $y$. Evidently $x \neq z_{1}$. If $K$ consists of a single point or if $M-x y$ is vacuous, we may arrive at a contradiction easily by using the Lemma. If neither of these two hold, let $S$ be the set of all points $\left[P_{x d}\right]$ such that $d$ contains a point of $K$. The arc $x y$ contains a point $z_{2}$ such that either (a) $z_{2}$ belongs to $S$ and every point of $S$ lies on the subarc $z_{2} y$ of $x y$, or (b) $z_{2}$ belongs to $x y-S$, every point of $S$ lies on the subarc $z_{2} y$ of $x y$ and $z_{2}$ is a limit point of $S$, or (c) $S$ is vacuous and $z_{2}=z_{1}$. Since $x$ does not belong to $M(K), x$ is not a point of $S$. If $x$ is a limit point of $S$, it is also a limit point of $K \dagger$ which is contrary to the hypothesis. Then $z_{2} \neq x$. Let $z_{3}$ be the first point of the set $z_{1}+z_{2}$ on the arc $x y$ in the order $x$ to $y$. If $z_{3}=y$, we may obtain a contradiction by using the Lemma. Then $x \neq z_{3} \neq y$.

Suppose $<x z_{3}$ contains a point $p$ such that $M-x y$ contains no component $d$ such that $p$ lies between $P_{x d}$ and $P_{y d}$. If $x$ and $y$ lie in the same

* A set $K$ is said to be a component of a set $H$, if $K$ is a connected subset of $B$ and there is no connected subset of $H$ which contains $K$ as a proper subset.

$\dagger$ Since only a finite number of the components of $M-x y$ are of diameter greater than any given positive number. Cf. my paper, Note on a theorem concerning continuous curves, Annals of Mathematics, (2), vol. 28 (1927), pp. 501-2. 
component of $M-p$, there exists an arc $\alpha$ with end points $x$ and $y$ and lying entirely in the set $M-p .^{*}$ In the order from $x$ to $y$ the arc $\alpha$ has a last point $t_{1}$ in common with the subarc $x p$ of the arc $x y$; and on the subarc $t_{1} y$ of $\alpha$ there is a first point $t_{2}$ in common with the subarc $p y$ of $x y$. If $d_{\alpha}$ denotes the component of $M-x y$ containing the subarc $t_{1} t_{2}$ of $\alpha$, the point $p$ lies between the points $P_{x d_{\alpha}}$ and $P_{y d_{\alpha}}$ contrary to our supposition that no such component exists. Hence

$$
M-p=M_{x}+M_{y}
$$

where $M_{x}$ and $M_{y}$ are non-vacuous mutually separated sets containing $x$ and $y$ respectively, and $M_{x}$ is connected. Then $M_{y}+p \supset K$, and by the Lemma, $x$ is not a limit point of $M(K)$, which contradicts the hypothesis of the theorem. Thus we have shown that if $p$ is any point of $\left\langle x z_{3}\right.$ there is a set $d_{p}$ such that $p$ lies between $P_{x d_{p}}$ and $P_{y d_{p}}$.

Since only a finite number of the components of $M-x y$ are of diameter greater than a given positive number, there is a component $d_{1}$ such that (a) $z_{3}$ lies between $P_{x d_{1}}$ and $P_{y d_{1}}$ on the arc $x y$, (b) if $d$ is any component of $M-x y$ such that $z_{3}$ lies between $P_{x d}$ and $P_{y d}$ on the arc $x y$, then $P_{x d}$ lies on the subarc $y P_{x d_{1}}$. If $P_{x d_{1}} \neq x$, there is a component $d_{2}$ of $M-x y$ such that (a) $P_{x d_{1}}$ lies between $P_{x d_{2}}$ and $P_{y d_{2}}$, (b) if $d$ is any component of $M-x y$ such that $P_{x d_{1}}$ lies between $P_{x d}$ and $P_{y d}$, then $P_{x d}$ lies on the subarc $y P_{x d_{2}}$ of the arc $x y$. Continue this process. In general, if $P_{x d_{i-1}} \neq x$, there is a component $d_{i}(i>1)$ of $M-x y$ such that (a) $P_{x d_{i-1}}$ lies between $P_{x d_{i}}$ and $P_{y d_{i}}$ on the arc $x y$, (b) if $d$ is any component of $M-x y$ such that $P_{x d_{i-1}}$ lies between $P_{x d}$ and $P_{y d}$, then $P_{x d}$ lies on the subarc $y P_{x d_{i}}$ of the arc $x y$. If for any value of $i, P_{x d_{i}}=x$, the process terminates at this point; if not, we continue the process indefinitely.

In the set $z_{3} P_{y d_{1}}>$ there is a point $z_{0}$ such that either (1) there is a component $d_{0}$ of $M-x y$ containing a point $z$ of $K$ such that $P_{x d_{0}}=z_{0}$, or (2) $z_{0}$ is a point of $K$. In case (1), by a theorem due to R. L. Wilder, $\dagger$ there exists an arc $A_{0}$ whose end points are $z$ and $z_{0}$ and such that $A_{0}$ belongs to $d_{0}$ except for the point $z_{0}$. In case (2), let $A_{0}$ and $z$ each denote the single point $z_{0}$. For every $i>0$ such that $d_{i}$ is defined, there exists an $\operatorname{arc} A_{i}$ with end points $P_{x d_{i}}$ and $P_{y d_{i}}$ and such that $d_{i}$ contains $\left\langle A_{i}\right\rangle$.

If there exists an integer $n$ such that $P_{x d_{n}}=x$ and $n$ is odd, let

* Cf. R. L. Moore, Concerning continuous curves in the plane, Mathematische Zeitschrift, vol. 15 (1922), p. 255, Theorem 1.

† Concerning continuous curves, Fundamenta Mathematicae, vol. 7 (1925), Theorem 1, p. 342. 


$$
\begin{aligned}
\alpha_{1}=A_{0}+z_{0} P_{y d_{2}}+A_{2}+P_{x d_{2}} P_{y d_{4}} & +A_{4}+\cdots+P_{x d_{z i-2}} P_{y d_{z i}} \\
& +A_{z i}+\cdots+A_{n-1}+P_{x d_{n+1}+1} x \\
\alpha_{2}=y P_{y d_{1}}+A_{1}+P_{x d_{1}} P_{y d_{z}}+ & A_{z}+\cdots+P_{x d_{2 i-1}} P_{y d_{z i+1}} \\
& +A_{z i+1}+\cdots+P_{x d_{n-2}} P_{y d_{n}}+A_{n} .
\end{aligned}
$$

It is evident that $\alpha_{1}$ and $\alpha_{2}$ are arcs with end points $z$ and $x$ and $y$ and $x$ respectively, and that they have only the point $x$ in common. Then $\alpha_{1}+\alpha_{2}$ is an arc of $M$ from $y$ to $z$ containing the point $x$. If there exists an integer $n$ such that $P_{x d_{n}}=x$ and $n$ is even, let

$$
\begin{aligned}
\alpha_{1}=A_{0}+z_{0} P_{y d_{2}}+A_{2}+P_{x d_{2}} P_{y d_{4}} & +A_{4}+\cdots+P_{x d_{2 i-2}} P_{y d_{2 i}} \\
& +A_{2 i}+\cdots+P_{x d_{n-2}} P_{y d_{n}}+A_{n}, \\
\alpha_{2}=y P_{y d_{1}}+A_{1}+P_{x d_{1}} P_{y d_{3}}+ & A_{3}+\cdots+P_{x d_{z i-1}} P_{y d_{2 i+1}} \\
+ & A_{2 i+1}+\cdots+A_{n-1}+P_{x d_{n-1}} x .
\end{aligned}
$$

In this case we see that $\alpha_{1}+\alpha_{2}$ is an arc of $M$ from $z$ to $y$ which contains the point $x$.

If for every integer $n, P_{x d_{n}} \neq x$, there is a point $q$ which is the sequential limit point of the sequence $P_{x d_{1}}, P_{x d_{2}}, P_{x d_{3}}, \cdots$. We shall show that $q=x$. If not, then there is a component $d_{q}$ of $M-x y$ satisfying the conditions (a) and (b) with respect to the point $q$. There is a smallest integer $j$ such that $P_{x d_{j}}$ is a point of $q P_{y d_{q}}>$. Then $d_{j+1}$ fails to satisfy condition (b), since $P_{x d_{j}}$ lies between $P_{x d_{q}}$ and $P_{y d_{q}}$ on the arc $x y$ but the arc $y P_{x d_{j+1}}$ does not contain $P_{x d_{q}}$. Hence $q=x$. In this case let

$$
\begin{aligned}
& \alpha_{1}=A_{0}+z_{0} P_{y d_{2}}+\sum_{i=1}^{\infty}\left(A_{2 i}+P_{x d_{2 i}} P_{y d_{2 i+2}}\right), \\
& \alpha_{2}=y P_{y d_{1}}+\sum_{i=1}^{\infty}\left(A_{2 i-1}+P_{x d_{z i-1}} P_{y d_{2 i+1}}\right) .
\end{aligned}
$$

Since $x$ is the sequential limit point of the sequences $\left[P_{x d_{2 i}}\right]$ and $\left[P_{x d_{2 i-1}}\right]$ and the diameters of the $\operatorname{arcs} A_{i}$ approach zero as $i$ increases, the sets $\alpha_{1}$ and $\alpha_{2}$ are $\operatorname{arcs}$ of $M$ with end points $z$ and $x$ and $y$ and $x$ respectively. It is evident that the arcs $\alpha_{1}$ and $\alpha_{2}$ have only the point $x$ in common, and thus $\alpha_{1}+\alpha_{2}$ is an $\operatorname{arc}$ of $M$ with end points $z$ and $y$ and containing the point $x$.

In any case then, the point $x$ lies on some arc of $M$ whose end points $y$ and $z$ belong to $K$. But this is contrary to the assumption that $x \subset \overline{M(K)}$ $-M(K)$, which proves our theorem. 
THEOREM 3. If $K$ is any subset of the continuous curve $M$ and $\alpha$ is an arc of $M$ whose end points belong to $\overline{M(K)}$, the arc-curve $M(K)$ contains every point of $\alpha$ except possibly the end points.

Suppose there exists an arc $\alpha$ of $M$ whose end points belong to $\overline{M(K)}$ such that $\langle\alpha\rangle$ contains a point $z$ that does not belong to $M(K)$. Since the end points of $\alpha$ belong to $\overline{M(K)}$, the set $K$ contains at least two points. Since $z$ belongs to $\langle\alpha\rangle$, it is not an end point of $M .^{*}$ Hence either (a) $z$ is a cut point of $M$ or (b) $z$ lies on some simple closed curve of $M$. $\dagger$ Suppose $z$ is a cut point of $M$. As $M(K)$ is connected and does not contain $z$, there is a component $H$ of $M-z$ which contains $M(K)$. As $\langle\alpha\rangle$ contains $z$, the point $z$ is not an end point of the continuous curve $H+z$; and as $H$ is connected, $z$ is not a cut point of $H+z$. Hence $z$ lies on a simple closed curve of $H+z$. Then in either case $z$ lies on a simple closed curve $J$ of $M$, and if $z$ is a cut point of $M$, the set $J-z$ lies in the component of $M-z$ containing $M(K)$.

By a theorem due to G. T. Whyburn $\ddagger$ there is a maximal cyclic curve $C$ of $M$ containing the simple closed curve $J$. If $C$ contains two points $x$ and $y$ of $K$, let $J_{x z}$ be a simple closed curve of $C$ containing $x$ and $z$. If the point $y$ lies on $J_{x z}$, there is an arc of $J_{x z}$ with end points $x$ and $y$ and containing $z$. Suppose $y$ does not belong to $J_{x z}$. Now as $x$ is not a cut point of $C, \S$ there exists an arc $y v z$ of $C-x$ with end points $y$ and $z$. In the order from $y$ to $z$ let $t$ be the first point of $J_{x z}$ on the arc $y v z$. If $t \neq z$, then the subarc $y t$ of $y v z$ plus that arc of $J_{x z}$ from $t$ to $x$ that contains $z$ is an arc of $C$ from $x$ to $y$ containing $z$; while if $t=z$, either $\operatorname{arc}$ of $J_{x z}$ from $x$ to $z$ may be used together with the subarc $y t$ of $y v z$. Then if $C$ contains two points $x$ and $y$ of $K$, there is an arc of $C$ with end points $x$ and $y$ and containing $z$; and thus $z$ belongs to $M(K)$. Now suppose $C$ contains just one point $x$ of $K$. Then some component $D_{y}$ of $M-C$ contains a point $y$ of $K$. The component $D_{y}$ has just one limit

- Cf. W. L. Ayres, Concerning continuous curves and correspondences, Annals of Mathematics, (2), vol. 28 (1927), pp. 396-418, Theorem 4, and G. T. Whyburn, Concerning continua in the plane, these Transactions, vol. 29 (1927), pp. 369-400, Theorem 12.

$\dagger$ W. L. Ayres, Concerning continuous curves and correspondences, loc. cit., Theorem 3, and G. T. Whyburn, loc. cit., Theorem 22.

$\ddagger$ Cyclicly connected continuous curves, Proceedings of the National Academy of Sciences, vol. 13 (1927), pp. 31-38, Theorem 3. A continuous curve $M$ is said to be cyclicly connected if every two points of $M$ lie together on some simple closed curve of $M$. A cyclicly connected continuous curve $C$, which is a subset of a continuous curve $M$, is said to be a maximal cyclic curve of $M$ if $M$ contains no cyclicly connected continuous curve of which $C$ is a proper subset.

No point of a cyclicly connected continuous curve is a cut point of the curve. See G. T. Whyburn, Cyclicly connecled continuous curves, loc. cit. 


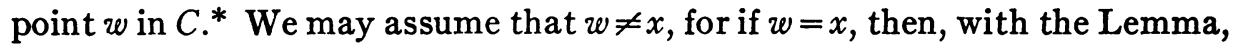
we could find another component of $M-C$ containing a point of $K$ and whose limit point in $C$ is not $x$. Since $M(K)$ is a connected subset of $M-z$, the points $w$ and $z$ are distinct. There exists an arc $\alpha_{1}$ with end points $w$ and $y$ and lying in $D_{y}$ except for the point $w$. As above there exists an $\operatorname{arc} \alpha_{2}$ of $C$ with end points $w$ and $x$ and containing the point $z$. The set $\alpha_{1}+\alpha_{2}$ is an arc of $M$ containing $z$ and whose end points $x$ and $y$ belong to $K$. Hence $z$ belongs to $M(K)$. Finally we suppose that $C$ contains no point of $K$. Let $[D]$ be the set of all components of $M-C$ that contain points of $K$, and let $[P]$ be the set of all points of $C$ such that each point $P$ is the limit point of one of the components of $[D]$. If $[P]$ consists of just one point $P$, then $P$ is a cut point of $M$, and let

$$
M-P=M_{1}+M_{2},
$$

where $M_{1}$ and $M_{2}$ are non-vacuous mutually separated sets and $M_{1}$ is that component of $M-P$ containing $C-P$. Evidently $z \neq P$, for $M(K)$ and $J-P$ lie in different components of $M-P$. Then $\overline{M(K)} \subset M_{2}+P$ and, by the Lemma, $z$ cannot belong to the $\operatorname{arc} \alpha$. Thus $[P]$ contains at least two points $u_{1}$ and $u_{2}$. Let $D_{i}(i=1,2)$ be a component of $[D]$ with $u_{i}$ as its limit point. The set $D_{i}$ contains a point $v_{i}$ of $K$. There exists an $\operatorname{arc} \beta_{i}$ whose end points are $u_{i}$ and $v_{i}$ and which lies in $D_{i}$ except for $u_{i}$. There exists an $\operatorname{arc} \beta_{3}$ of $C$ with end points $u_{1}$ and $u_{2}$ and containing $z$. The set $\beta_{1}+\beta_{2}+\beta_{3}$ is an $\operatorname{arc}$ of $M$ containing $z$ whose end points $v_{1}$ and $v_{2}$ belong to $K$. Hence $z$ belongs to $M(K)$. Thus the assumption that $z$ does not belong to $M(K)$ leads to a contradiction in every case.

In Theorem 3 we see that every interior point of an $\operatorname{arc}$ of $M$, whose end points belong to $\overline{M(K)}$, belongs to $M(K)$. We may ask if the end points must belong to $M(K)$ also. The following simple example shows that this is not the case. Let $M$ be the interval whose end points are $(0,0)$ and $(1,0)$. Let $K$ be the segment, i.e. the interval minus its end points, from $(0,0)$ to $(1,0)$. The points $(0,0)$ and $(1,0)$ are points of $\overline{M(K)}-M(K)$, and every interior point of the arc of $M$ joining them is a point of $M(K)$.

TheOREM 4. If $M$ is a continuous curve and $K$ is a subset of $M$, the set $\overline{M(K)}$ is identical with the arc-curve $M(\bar{K})$.

By Theorem 1, $M(K) \subset M(\bar{K})$; and by Theorem 2,

$$
\overline{M(K)}-M(K) \subset \bar{K}-K .
$$

* G. T. Whyburn, Cyclicly connected continuous curves, loc. cit., Theorem 2. 
Hence $\overline{M(K)}=M(K)+\overline{(M(K)}-M(K)) \subset M(\bar{K})+(\bar{K}-K)=M(\bar{K})$. Since $K \subset M(K), \bar{K} \subset \overline{M(K)}$. By Theorem $1, M(\bar{K}) \subset M(\overline{M(K)})$. But by Theorem 3, $M(\overline{M(K)})=\overline{M(K)}$. As $\overline{M(K)} \subset M(\bar{K}) \subset M(\overline{M(K)})=M(K)$, we have $\overline{M(K)}=\boldsymbol{M}(\bar{K})$.

THEOREM 5. If $K$ is any subset of the continuous curve $M$, the set $M(K)$ is arc-wise connected im kleinen. ${ }^{*}$

Let $x$ be any point of $M(K)$, and $\epsilon$ be any positive number. Since $M$ is a continuous curve, there exists a positive number $\delta_{e x}$ such that any point of $M$ whose distance from $x$ is less than $\delta_{\epsilon x}$ can be joined to $x$ by an $\operatorname{arc}$ of $M$ whose diameter is less than $\epsilon$. Let $y$ be any point of $M(K)$ whose distance from $x$ is less than $\delta_{e x}$. There exists an $\operatorname{arc} \alpha$ of $M$ with end points $x$ and $y$ and whose diameter is less than $\epsilon$. By Theorem 3, every point of $\alpha$ belongs to $M(K)$, and thus $M(K)$ is arc-wise connected im kleinen.

From Theorems 4 and 5, we have

THEOREM 6. If $K$ is any subset of the continuous curve $M$, the set $\overline{M(K)}$ is connected im kleinen.

Since every point of a continuous curve which is not an end point is an interior point of some arc belonging to the continuous curve, we have from Theorems 3 and 6 the following result: If $K$ is any subset of the continuous curve $M$, every point of the set $\overline{M(K)}-M(K)$ is an end point of the continuous curve $\overline{M(K)}$.

From Theorems 2 and 5, we have the important result

THEOREM 7. If $K$ is a closed subset of the continuous curve $M$, the arccurve $M(K)$ is a continuous curve.

THEOREM 8. If $x$ and $y$ are distinct points of the continuous curve $M$, neither $x$ nor $y$ is a cut point of the arc-curve $M(x+y)$.

Every point $z$ of $M(x+y)$ lies on some $\operatorname{arc} A_{z}$ of $M$ whose end points are $x$ and $y$. The subarc $y z$ of $A_{z}$ is a subset of $M(x+y)-x$. As each point of $M(x+y)-x$ may be joined to $y$ by an arc which belongs to $M(x+y)-x$, the set $M(x+y)-x$ is connected. Similarly $M(x+y)-y$ is connected. Hence neither $x$ nor $y$ is a cut point of $M(x+y)$.

The following example shows that Theorem 8 does not remain true if the set $K$ contains more than two points. Let $M$ be the interval from $(0,0)$

* A point set $B$ is said to be arc-wise connected im kleinen if for each point $x$ of $H$ and each positive number $\epsilon$, there exists a positive number $\delta_{\epsilon x}$ such that any point of $B$ whose distance from $x$ is less than $\delta_{\epsilon x}$ can be joined to $x$ by an arc of $H$ whose diameter is less than $\epsilon$. For closed sets the properties connected im kleinen and arc-wise connected im kleinen are equivalent. 
to $(2,0)$. Let the set $K$ consist of the three points $(0,0),(1,0)$, and $(2,0)$. In this case the point $(1,0)$ is a cut point of the arc-curve $M(K)$.

Theorem 9. If $K$ is any subset of the continuous curve $M$, then (1) if $P$ is a cut point of $M(K)$ every component of $M(K)-P$ contains at least one point of $K,(2)$ every cut point of $M(K)$ is a cut point of $M$.

Part (1) is an application of the Lemma. For Part (2), let $P$ be a cut point of $M(K)$ and $x$ and $y$ points of $M(K)-P$ which do not lie in the same component of $M(K)-P$. If $M-P$ is connected, there is an $\operatorname{arc} \alpha$ of $M-P$ with end points $x$ and $y .{ }^{*}$ By Theorem 3, every point of $\alpha$ belongs to $M(K)$. Hence $x$ and $y$ belong to the same component of $M(K)-P$, contrary to hypothesis. Then every cut point of $M(K)$ is also a cut point of $M$.

Theorem 10. If $K$ is a closed subset of the continuous curve $M$, then (1) every end point of $M(K)$ belongs to $K,(2)$ if $T$ is a maximal connected subset of $M-M(K)$, the set $T$ has only one limit point in $M(K),(3)$ if $D$ is a bounded complementary domain of $M(K)$ such that no point of $K$ is interior to the outer boundary of $D \dagger$, then the boundary of $D$ is a simple closed curve, (4) if $D$ is a complementary domain of $M(K)$ there is a complementary domain $D_{1}$ of $M$ which is a subset of $D$ and such that every boundary point of $D$ is also a boundary point of $D_{1}$.

Part (1). If $P$ is a point of $M(K)-K$, there exists an arc of $M$ whose end points are points of $K$ and containing $P$ as an interior point. Then $P$ cannot be an end point of $M(K)$ by Theorem 4 of my paper Concerning continuous curves and correspondences. $\ddagger$

Part (2). Suppose $T$ has two limit points $P$ and $Q$ in $M(K)$. Using a theorem due to R. L. Wilder, $\S$ there exists an arc whose end points are $P$ and $Q$ and which lies entirely in $T$ except for the points $P$ and $Q$. But this is seen to be impossible from Theorem 3 .

Part (3) may be proved using a theorem due to R. L. Moore.\|

Part (4) may be proved without difficulty using Part (2) and the result of my paper Note on a theorem concerning continuous curves. I

- Cf. R. L. Moore, loc. cit.

† Cf. R. L. Moore, loc. cit., for definition of outer boundary and theorems concerning it.

$\ddagger$ Loc. cit.

Loc. cit.

II Concerning paths that do not separate a given continuous curve, Proceedings of the National Academy of Sciences, vol. 12(1926), pp. 745-753, Theorem 6.

ILoc. cit. 
II

Definition. A subset $K$ of a continuous curve $M$ will be called a basic set of the continuous curve if $M(K)=M$. A basic set of a continuous curve $M$ will be said to be irreducible if it contains no proper subset which is a basic set of $M$.

THEOREM 11. In order that a subset $K$ of a continuous curve $M$ be a basic set of $M$, it is necessary and sufficient that (1) $K$ contain at least two points unless $M$ consists of a single point, (2) $K$ contain every end point of $M,(3)$ if $P$ is a cut point of $M, K$ contain a point of every component of $M-P$.

The conditions are necessary. The necessity of the first condition is obvious. The necessity of the second and third conditions follows from Theorem 10, Part (1), and Theorem 9, Part (1), respectively, since we assume $M(K)=M$.

The conditions are sufficient. Let $K$ be a subset of $M$ satisfying the three conditions, and let us suppose that $M-M(K)$ is not vacuous. Let $x$ be any point of $M-M(K)$ and $y$ a point of $M(K)$, and let $x y$ denote an arc of $M$ with end points $x$ and $y$. Let $z$ denote the first point of $\overline{M(K)}$ on the arc $x y$ in the order $x$ to $y$.

Suppose $x$ is not a limit point of $M(K)$. Then the points $x$ and $z$ are distinct. If $z$ is a cut point of $M$, let $M_{1}$ be the component of $M-z$ containing $x$, and $M_{2}$ be some other component of $M-z$. By condition (3), $M_{1}$ and $M_{2}$ contain points $e_{1}$ and $e_{2}$ of $K$. There exists an arc $\alpha_{1}$, whose end points are $x$ and $e_{1}$ and every point of which belongs to $M_{1}$, and an arc $\alpha_{2}$, whose end points are $e_{2}$ and $z$ and such that every point of $\alpha_{2}$ except $z$ is a point of $M_{2}$. Let $p$ be the first point of the subarc $x z$ of the $\operatorname{arc} x y$ on the $\operatorname{arc} \alpha_{1}$ in the order $e_{1}$ to $x$. Since $\alpha_{1} \subset M_{1}, p \neq z$. Then the subarc $e_{1} p$ of $\alpha_{1}$ plus the subarc $p z$ of $x y$ plus the $\operatorname{arc} \alpha_{2}$ is an arc of $M$ whose end points $e_{1}$ and $e_{2}$ belong to $K$. Then every point of the subarc $p z$ of $x y$ belongs to $M(K)$. But every point of the set $p z>$ precedes $z$ on the arc $x y$ in the order $x$ to $y$, which contradicts our assumption that $z$ is the first point of the set $\overline{M(K)}$ on this arc in this order.

If $z$ is not a cut point of $M$, let $w$ be a point of $K$ distinct from $z$. The existence of the point $w$ follows from condition (1). As $M-z$ is connected, there is an arc $x w$ with end points $x$ and $w$, every point of which belongs to $M-z$. In the order $x$ to $w$ let $q_{1}$ be the last point of the subarc $x z$ of $x y$ on the arc $x w$, and let $q_{2}$ be the first point of the set $\overline{M(K)}$ on the subarc $q_{1} w$ of $x w$. We have $q_{2} \neq q_{1} \neq z$, and thus the subarc $q_{2} q_{1}$ of $x w$ plus the subarc $q_{1} z$ of $x y$ is an arc of $M$ which has only its end points in common with $\overline{M(K)}$. But this is impossible by Theorem 3. 
Hence every point $x$ of $M-M(K)$ is a limit point of $M(K)$, and thus of $K$ (Theorem 2). As no point of $M-M(K)$ is an end point of $M$ (Condition (2)), the point $x$ is either a cut point of $M$ or lies on some simple closed curve $J$ of $M^{*}$ If $x$ is a cut point of $M$, let $M_{1}$ and $M_{2}$ be distinct components of $M-x$, and let $e_{1}$ and $e_{2}$ be points of $K$ which belong to $M_{1}$ and $M_{2}$ respectively. Every arc of $M$ with end points $e_{1}$ and $e_{2}$ contains $x$. Hence $x$ belongs to $M(K)$, contrary to assumption. If $x$ lies on a simple closed curve $J$ of $M$, let $y$ and $z$ be points of $J$ distinct from $x$ and from each other, and let $y x z$ denote the arc of $J$ from $y$ to $z$ which contains the point $x$. The points $y$ and $z$ belong to $\overline{M(K)}$, since we have shown above that $\overline{M(K)}=M$. Then the point $x$ belongs to $M(K)$, since $y x z$ is an arc of $M$ whose end points belong to $\overline{M(K)}$. Therefore the conditions are sufficient.

THEOREM 12. In order that a basic set $K$ of a continuous curve $M$ be irreducible, it is necessary and sufficient that there should not exist an arc of $M$ which contains three distinct points of $K$.

The condition is necessary. Suppose $K$ is an irreducible basic set of $M$, and that $M$ contains an $\operatorname{arc} \alpha$ which contains three distinct points $x, y, z$ of $K$. One of these points, and we will suppose $x$ is the one, lies between the other two on the arc $\alpha$. We shall prove that $K-x$ is a basic set of $M$ and thus show that $K$ is reducible, by showing that $K-x$ satisfies the three conditions of Theorem 11.

The set $K-x$ satisfies condition (1) since it contains the two points $y$ and $z$. The set $K$ contains every end point of $M$ since it is a basic set of $M$, and $x$ is not an end point of $M$ since it is interior to an arc of $M$, namely the arc $\alpha$. Hence $K-x$ contains every end point of $M$ and condition (2) is satisfied. If $K-x$ does not satisfy condition (3), there is a cut point $P$ of $M$ and a component $M_{1}$ of $M-P$ which contains no point of $K-x$. Since $K$ satisfies condition (3), $M_{1}$ contains the single point $x$ of $K$. Then, by the Lemma, the point $x$ cannot be interior to the subarc $y z$ of $\alpha$. Thus $K-x$ satisfies all three conditions of Theorem 11 and is a basic set of $M$.

The condition is sufficient. Suppose $K$ contains a proper subset $H$ which is a basic set of $M$. Let $x$ be a point of $K-H$. Since $x \subset M-H=M(H)-H$, there exists an $\operatorname{arc} \beta$ of $M$, which contains $x$ and whose end points $y$ and $z$ are points of $H$. Then the $\operatorname{arc} \beta$ of $M$ contains three distinct points $x, y$ and $z$ of $K$.

THEOREM 13. In order that a basic set $K$ of a continuous curve $M$ be irreducible it is necessary and sufficient that (1) $K$ contain no cut point of $M$,

* See my paper, Concerning continuous curves and correspondences, loc. cit., Theorem 3. See also G. T. Whyburn, Concerning continua in the plane, loc. cit., Theorem 22. 
(2) if $C$ is a maximal cyclic curve of $M$, the set $C$ contain two points, one point or no point of $K$ according as $C$ contains no cut point, one cut point or more than one cut point of $M$.

The conditions are necessary. Let $K$ be an irreducible basic set of the continuous curve $M$. If $K$ contains a point $z$ which is a cut point of $M$, let $M_{1}$ and $M_{2}$ be two distinct components of $M-z$. Since $K$ is a basic set, by Theorem 11 the component $M_{i}(i=1,2)$ contains a point $x_{i}$ of $K$. Let $\alpha$ denote an arc of $M$ whose end points are $x_{1}$ and $x_{2}$. Since $M_{1}$ and $M_{2}$ are components of $M-z$, the arc $\alpha$ contains the point $z$. Then $K$ is not irreducible by Theorem 12 .

If $C$ is a maximal cyclic curve of $M$ and contains no cut point of $M$, then $C=M{ }^{*}$ Then $K$ consists of just two points, for if $K$ contains three or more points, there exists an $\operatorname{arc}$ of $M$ containing three points of $K \dagger$ and, by Theorem 12, the set $K$ is not an irreducible basic set. If $C$ contains just one cut point $P$ of $M$, the set $C-P$ is a component of $M-P$ and thus contains at least one point of $K$ by Theorem 11. If $C-P$ contains two points $x$ and $y$ of $K$, there is an arc $\alpha_{1}$ belonging to $C$ which contains $y$ and whose end points are $P$ and $x$. Let $M_{1}$ be a component of $M-P$ which is distinct from $C-P$, and let $z$ be a point of $K$ which belongs to $M_{1}$ (Theorem 11). There exists an $\operatorname{arc} \alpha_{2}$ whose end points are $P$ and $z$ and which lies in $M_{1}$ except for the point $P$. Then $\alpha_{1}+\alpha_{2}$ is an arc of $M$ which contains three points $x, y, z$ of $K$. But this is impossible since $K$ is an irreducible basic set (Theorem 12). If $C$ contains two cut points $P$ and $Q$ of $M$, let $M_{1}$ be a component of $M-P$ which does not contain $C-P$, and let $M_{2}$ be a component of $M-Q$ which does not contain $C-P$. It may be seen easily that $M_{1}$ and $M_{2}$ have no common points. Let $x$ and $y$ be points of $K$ which belong to $M_{1}$ and $M_{2}$ respectively (Theorem 11). Now suppose $C$ contains a point $z$ of $K$. Since condition (1) is necessary, $P \neq z \neq Q$. There exist $\operatorname{arcs} \alpha_{1}$ and $\alpha_{2}$ with end points $x$ and $P$ and $y$ and $Q$ respectively, and such that $\alpha_{1}$ lies in $M_{1}$ except for the point $P$ and $\alpha_{2}$ lies in $M_{2}$ except for the point $Q$. There exists an $\operatorname{arc} \alpha_{3}$ with end points $P$ and $Q$ and such that

$$
z \subset \alpha_{3} \subset C \text {. }
$$

Then $\alpha_{1}+\alpha_{2}+\alpha_{3}$ is an arc of $M$ containing three points $x, y$, and $z$ of $K$. But this is impossible, for $K$ is an irreducible basic set of $M$.

* Cf. G. T. Whyburn, Cyclicly connected continuous curves, loc. cit., Theorem 1.

$\dagger$ In the proof of Theorem 3 we showed that if $x, y$ and $z$ are any three points of a cyclicly connected continuous curve $C$, there is an arc of $C$ with end points $x$ and $y$ and containing $z$. 
The conditions are sufficient. Let $K$ be a basic set of $M$ satisfying the two conditions, and suppose that $K$ is reducible. Then $K$ contains a proper subset $H$ which is a basic set of $M$. Let $x$ be a point of $K-H$. By Theorem 11, the set $H$ contains every end point of $M$. Then the point $x$ is not an end point of $M$. By condition (1), the set $K$ contains no cut point of $M$; and since $x \subset K$, the point $x$ is not a cut point of $M$. Hence the point $x$ must lie on some simple closed curve of $M$, and there is a maximal cyclic curve $C$ containing the point $x .^{*}$ If $C$ contains no cut point of $M$, every point of $H$ belongs to $C$. Since $H$ contains at least two points (Theorem 11) and $H \subset K$, the maximal cyclic curve $C$ contains three points of $K$, contrary to condition (2). If $C$ contains one cut point $P$ of $M$, the set $C-P$ is a component of $M-P$ and contains at least one point of $H$ (Theorem 11). Then the curve $C$ contains two points of $K$, contradicting condition (2). If $C$ contains more than one cut point of $M, C$ contains the point $x$ of $K$, thus contradicting condition (2). Hence the conditions are sufficient.

It is evident that every basic set of a bounded continuous curve contains a subset which is an irreducible basic set. Also there are continuous curves which have more than one irreducible basic set.

* Cf. G. T. Whyburn, Cyclicly connected continuous curves, loc. cit.

The University or Pennsylvania, Philadelphia, Pa. 